\title{
Solutions to improve the decision-making process for dynamic allocation of resources in military pre-conflict environments
}

\author{
Ioan-Danut Balos ${ }^{1, *}$, Catalin Cioaca ${ }^{2}$, and Mircea Boscoianu ${ }^{1}$ \\ ${ }^{1}$ Transilvania University of Brasov, Department of EIM, 5 M. Viteazul, Brasov, Romania \\ 2 "Henri Coanda" Air Force Academy, FSM Department, 160 M. Viteazul, Brasov, Romania
}

\begin{abstract}
The complexity of the international security environment compels us to analyse the dynamics of the civil-military decision making process regarding the optimal allocation and efficient use of resource units (UoR). The main purpose of this paper is to improve the civil-military decision-making process by analysing from a dynamic perspective the influence of uncertainty on UoR allocation, thus providing a fast intuitive image of resource ratio. To achieve this goal, risk and uncertainty are defined in terms of knowledge (total or partial) of possible future events or potential outcomes of the action/ decision. The decision alternatives capitalize the results obtained by the optional (the real options analysis in conditions of uncertainty) and flexibility (the prediction of transition moment according to the life cycle of resources). Moreover, these findings could have a positive effect across the full range of economic sectors.
\end{abstract}

\section{Introduction}

From a strategic point of view the complexity of the international security environment compels us to analyze the dynamics of the civil-military decision making regarding the optimal allocation and efficient use of resource units (UoR). The statistical analysis of conflict shows that a non-conflict state can be considered a special case of the conflict state in the same way as peace is a preliminary stage that accumulates future confrontations sources [1]. Currently, according to Armed Conflict Database, there are 9 ongoing global conflicts of high intensity, 19 of medium intensity and 13 low-intensity conflicts.

For this scientific approach, risk and uncertainty are defined in terms of total or partial knowledge of possible future events or potential outcomes of the action/ decision [2]. Regardless of the activity and the levels of applicability, decisions are characterized by the fact that they require time and effort, cause concern and anxiety and are a means of exerting influence.

The classical decision making process is based on the backward approach because it is trying to solve a problem with a chosen alternative before understanding what one might hope to gain by solving that problem [3]. The real options analysis (ROA) provides a

\footnotetext{
${ }^{*}$ Corresponding author: danut.balos@gmail.com
} 
theoretical justification of requests for additional UoR in a way that differs from the classical model (based on UoR flows).

Military decisions that seek to gain small and visible benefits with potentially negative and invisible side effects, lead to the disruption of a system that has survived just by using unpredictable shocks for continuous regeneration.

Disruption in the military system can take the form of actions with unintended consequences, repeated with a more sustained intensity in order to correct the side effects [4], which leads to disregarding the available resources or those that can be effectively mobilized in a timely manner.

The complex behavior of systems is a combination of "explosive" (the system contains only positive feedback, such as the emergence of a new weapons system) and "inert" (generated by the preponderance of negative feedback sites, such as defense spending) [5].

Each military system should answer the following questions: How are investments projected in order to have the necessary resources units available? How many units of resources are needed to take advantage of the first move (the initiative)? What are the solutions to minimize losses of units per resources? What are the upper and lower limits to investing in producing new units of resources?

The allocation of the resources process is used for reaching military strategic objectives through UoR distribution from the resource nexus, but the issue is generally presented in a quasi-static manner. Resource units which are the subject of military decision-making process usually include: weapons systems, categories/ types of forces, installation and network elements and defense research and development.

The main purpose of this paper is to improve the military decision making process by analyzing in a dynamic vision the influence of uncertainty on the UoR allocation. The model provides an intuitive image of the timing and intensity of investment transition, using binomial portfolio of real options.

\section{The cost-performance analysis in defence procurement}

The cost - performance analysis is specific to certain fields such as the airspace industry and defence industry, and is based on the hypothesis that the essential benefit are from the cost benefit analysis is given by performance in a wider sense.

The allocation of resources in order to reduce the risk of being surprised involves the development of risk assessment instruments whose results contribute to the identification of risk scenarios, the links between initial events and consequences, the probability of occurrence and mitigation measures [6].

Decision makers often, in the field of military operations, underestimate the risk of occurrence of initial events that have major impact and are difficult to predict [7].

At the strategic level, the objectives of the military action are influenced by political, military, economic and social risks. The most widely used risk assessment methods are focused on supporting the total military operation plan [8]. However, these techniques have a major limitation: the assumptions used by analysts can be highly subjective.

Risk management in defence procurement processes must be integrated with management requirements and configuration [9]. Thus, there have been identified four categories of risks (technical, financial, time and programmatic), having as main sources: changes in mission, technical inconsistencies, imposed budgets and tight deadlines [10]. Based on the results of the risk analysis, the level of confidence is established by estimated costs of the system associated with the lifecycle and the updated costs during the development of the acquisition program (Fig. 1). 


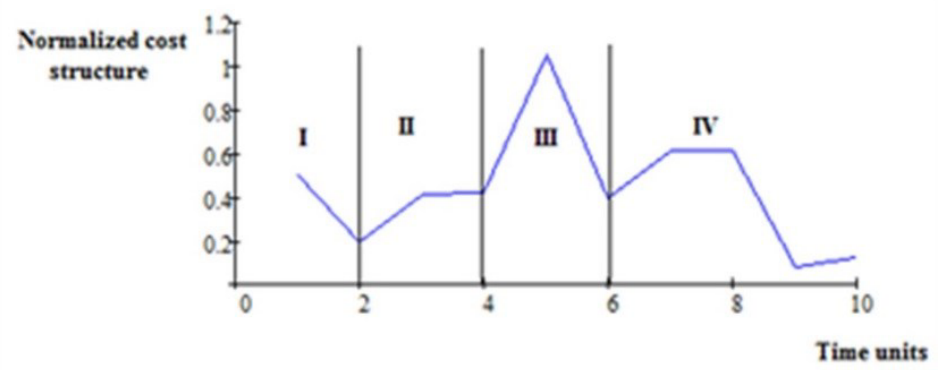

Fig. 1. The cost structure on lifecycle phases: I - studying and selecting concept; II - technological development; III - production and installation; IV - operating and support

The cost of the entire life cycle is structured as follows: I and II represent research and development costs; II and III correspond to acquisition costs/procurement expenses, IV represents operating costs and modernization. In terms of design, engineering, construction, testing, implementation and maintenance the acquisition of military equipment for endowment structures of forces requires a critical evaluation of the needs and capabilities of different available alternative systems and equipment from the opponent and associated costs. Modernization aims is to improve the operating performance, to cancel/ reduce the obsolescence, or to respond to new demands (by default it contains revitalizing and extending the resource).

The distribution of costs based on the lifecycle stages of military products differs from other products in that the duration and intensity of the consumption during certain stages are volatiles; also there are financial constraints as well as certain products characteristics, which all result in altering the altitude to investment on long term. Additionally, the various dynamics of evolution affects the strategic decision making process.

Cost assessments represent the input for the cost-performance analysis, which is developed in order to: identify the objectives of the mission and performance requirements; to support the performance allocation necessary for the functions of the system to work; to ensure selection of requirements regarding system design and to provide criteria for the selection of alternatives; to provide analytical confirmation that the project meets the end user requirements; to support system and process verification; to supports decisions on changes and upgrades throughout the lifecycle.

The real options approach is designed primarily to assist the management and investment selection in order to maximize the value of the organization [11-12]. Real options are completed and integrated solutions (using financial theory, economic analysis, management, decision theory, statistics and econometric modelling) aimed at improving decision making for military investment projects in the pre-crisis phase [13].

\section{Decision model development}

The need to optimize management strategies and to provide some flexibility to decision makers about the changes in the external environment of the organization should be based on the ingredient of optionality in order to quickly adapt to the actions (movements) of the competitors/ opponents.

Military decision makers constantly adapt and revise their strategies depending on the conditions of the operational environment. Also, it should not be overlooked the fact that the allocation of UoR is partially irreversible; some of them can easily be fully recovered, but others may only be partially recovered or even entirely lost. The regeneration resources 
process (from manufacturing to modernization) becomes a difficult process to assess in a conflictual environment.

For the development of a decision-making model, in which the use of UoR must become efficient, there the following assumptions are considered:

Hypothesis 1: two options are considered: the offensive (A), corresponding to the procurement cycle, which allows for the development of the capabilities needed for the achievement of the benefits from future opportunities; and delaying the decision of the offensive action (M), corresponding to the modernization cycle that allows for keeping the option open.

The option of going the offensive action encourages UoR allocation. In the case of irreversible allocations, there is an opportunity cost that represents the value of the postponing option. A and $\mathrm{M}$ have opposite effects, but they both contribute to increasing the level of uncertainty.

Hypothesis 2: for a dynamic options management, the envelope borders of the efficient use of a UoR nexus from weapons category are defined as follows: the lower limit corresponds to the NATO target of $2 \%$ of GDP for national defense; and the upper limit corresponds to Global Fire Power Index (GFPI). The upper limit also incorporates the technological progress. The study is designed for the complete lifecycle of a product.

Hypothesis 3: the increase in uncertainty is inversely proportional to the escalating stress and is a feature of the pre-conflict status. Currently, to manage resources, the military decision-making system is based on the concept of "military equipment static valuation", based on the achievement of some cumulative criteria necessary to the military equipment procurement programs that are subject to valuation, capitalization and depreciation [14].

The importance of strategic flexibility is given by the growing differences between solution and the classical model (e.g. the flexibility of the postponement decision when there is uncertainty regarding the future environmental conditions). If it would sacrifice option M (although UoR is usually irreversible), then the opportunity to make a specific decision "A" when new information is available would be lost. This mechanism has a significant impact on UoR timing allocation.

Hypothesis 4: the combination of effects of options A and $\mathrm{M}$ depend not only on their ratio $(\mathrm{W})$ within the binomial portfolio $\mathrm{P}(\mathrm{A}, \mathrm{M}, \mathrm{W})$, but also on the interactions between $\mathrm{A}$ and $\mathrm{M}$ on various levels of uncertainty.

Ideally, "W" should be 0 or 1 , but due to the inherent transition process, take values lying within the interval $(0,1)$, as follows: the variation $\mathrm{W}_{\mathrm{A}}$ and $\mathrm{W}_{\mathrm{M}}$ follows a logistic curve, while their sum any given time is 1 .

The concept of "portfolio" first appears in the context of these transition processes and is characterized by frictions and delays. The accumulative of UoR for offensive is not encouraged because the cost of delay option $\left(\mathrm{C}_{M}\right)$ increases simultaneously with the uncertainty.

The main interest is to identify the critical threshold for when UoR request appear [15]. The mechanism through which the uncertainties delay process of resource allocation disregards the fact that this allocation process could provide unpredicted possibilities for growth.

If the value of option A evolves simultaneously with uncertainty, the increase in uncertainty diminishes the critical threshold of UoR requests, thus inducing another type of relation between uncertainty and allocation of UoR. 


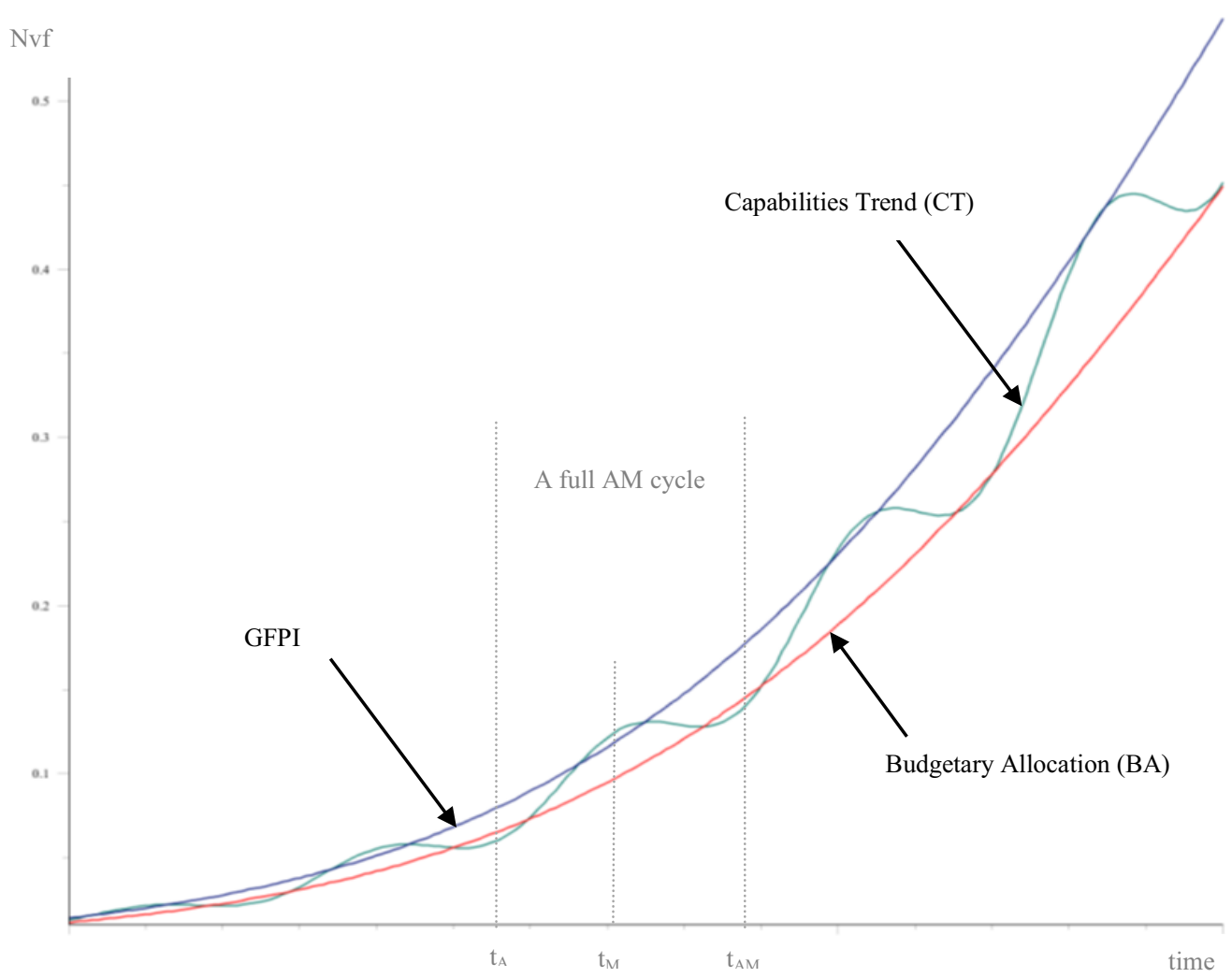

Fig. 2. The dynamics of the investments and military capabilities

In order to simplify the understanding of the current approach, the evolution of limitations can be approximated by means of a logistic function (or normal distribution function or a crisis evolution function). For the analyzed part, the functions corresponding to GFPI and budget allocations (BA) are defined in equation (1) and (2), and the evolution of military capability $\left(f_{C T}\right)$ in equation (3).

$$
\begin{gathered}
f_{G F P I}(t)=\frac{1}{2 \pi \sigma_{1}} e^{-\frac{\left(t-m_{1}\right)^{2}}{\left(\sigma_{1}\right)^{2}}} \\
f_{B A}(t)=\frac{1}{2 \pi \sigma_{2}} e^{-\frac{\left(t-m_{2}\right)^{2}}{\left(\sigma_{2}\right)^{2}}} \\
f_{C T}(t)=\operatorname{Re}\left(e^{-\frac{2 \pi t j}{t_{A M}-t_{A}}}\right) f_{d l}(t)+f_{m d}(t)
\end{gathered}
$$

In a graphical (Fig. 2) and intuitive representation of the normalized value of the analyzed functions (Nvf), the probable values of expectations $\left(m_{1}, m_{2}\right)$ and the standard deviation $\left(\sigma_{1}{ }^{2}, \sigma_{2}{ }^{2}\right)$ which quantify the dispersion of a specific data values sets, can be viewed as strategic objectives that incorporate a high degree of uncertainty. A completely ideal cycle $A M$, is characterized by duration $\left(t_{A M}-t_{A}\right)$, initial moment $\left(t_{A}\right)$ and endpoint $\left(t_{\mathrm{AM}}\right)$. The ratio expectation-variance determines the frequency of the decisions oscillations and thus, the duration of the AM cycle. The evolution of the military capabilities under this 
circumstances may be expressed by using the equation (3) which shows the dependence of these on the upper limitations (expected GFPI) and lower BA through the $f_{d l}(t)$ - the function of variance limitations (the width of the decision tunnel) and $f_{m d}(t)$ - the mean function of the limitations (trend achieved).

The immediate strategic choice between $\mathrm{A}$ and $\mathrm{M}$ has strong implications. A previous decision may position favorably the obtaining of an advantage and/ or of future resource savings.

\section{Conclusion}

As part of the planning processes, the volume, the structure and the way the resources are allocated for defence in order to fulfil the fundamental of defence and national objectives security are established.

The decision to launch the offensive action must be made either quickly in order to make use of the gained advantage, or later on when the environment becomes less uncertain.

Within the planning, programming, budgeting and evaluation system, the risks that may arise (respecting deadlines, procurement contracts, shortage of capabilities, necessary financial resources in the short / medium and long term, exterior condition) are taken into account and a reserve buffer-type reserve is provided in order to solve unexpected situations. Errors in this regard could result in failure to ensure the critical mass of defence resources for in a wider sense or unnecessary waste that could have been used for economic development. In this respect, it is imperative to create dynamic balance equilibrium able to fluidly support the necessary investments by means of multi-annual budgets.

The defence resources concept, as approached in a wider sense, including the correlation between budget allocation and capability requirements, as well as the exploitation of the results yielded by the engineering system within the phases of the procurement process management, represent important contributions to the dynamic planning of defences resource in volatile and turbulent environments.

The issue of the resources allocation was analysed based on the principles of complex engineering systems, allocation strategies design by integrating the concept of Dynamic Capabilities for Defence and correlating it with the option thinking concept expressed through the real options analysis paradigm.

Based on the results, the strategic decision-maker can better understand the spectrum of possible decisions and the mode of action in case of major shocks or turbulence. The method can be adapted at tactical level or over shorter periods of time, but the focus on the preparation and the preplanning sequences, should be maintained.

Future research directions include how operational and decision-making interferences comparatively evolve in multiple scenarios incorporating additional categories of resources.

\section{References}

1. H. Grotius, On the Law of War and Peace, (SPH, Bucharest, 1968)

2. T. B. Folta, J. P. O’Brien, Strat. Mgmt. J., 25, 121-138, (2004)

3. R. Keeney, Value-Focused Thinking: The Foundation for Decision Quality. (Available on: http://www.spe.org/ogf/print/subscribers/2012/08/08_12_OGF_10_Culture.pdf, 2012)

4. N. Taleb, Antifragile: Things That Gain from Disorder (Random House, New York, 2012)

5. B. Arthur, Complexity Economics: A Different Framework for Economic Thought, Complexity Economics, (Oxford Univ. Press, 2013)

6. R. Moraru, G. Babut, L.I. Cioca, Archives Of Mining Sciences 55 (4), 873-878, (2010) 
7. C. Cioaca, C.G. Constantinescu, M. Boșcoianu, R. Lile, Env. Eng. Manag. J., 14 (6), 1399-1408 (2015)

8. A. C. Karmperis, A. Sotirchos, I. Tatsiopoulos, K. Aravossis, J. of Comp. Model, 4 (1), 67-81 (2014)

9. T. J. Andersen, J. Manage. Stud., 41(8), 1271-1299 (2004)

10. N. Iancu, Contributii la dezvoltarea ingineriei sistemelor de armament terestru, (PhD Thesis, Military Technical Academy, Bucuresti, 2006)

11. F. Moreno-Hines, Real Options Applications for Public Sector Investment and Acquisitions, MITRE Technology Program (Available at: http://www.mitre.org/news/events/ tech07/1.html, 2007)

12. J. Mun, Real Options Analysis: tools and techniques for valuing strategic investments and decisions, (John Wiley and Sons, New Jersey, 2002)

13. C. Cioacă, M. Boscoianu, App. Mech. Mat., 436, 32-39 (2013)

14. *** Department of Defense (DoD), Financial Management Department of Defense Office of Inspector General September 30, 2005 Report on Development of the DoD Baseline for Military Equipment (D-2005-114), Office of Inspector General, Arlington (2005)

15. H. Smit, L. Trigeorgis, R\&D Manag., 36 (4), 403-419 (2006) 